\title{
VARIABILIDADE DA ESPESSURA DA LÂMINA FOLIAR EM DUAS ESPÉCIES DE LEUCAENA Benth. (LEGUMINOSAE) E SEUS HÍBRIDOS
}

\author{
VARIABILITY OF THE FOLIAR BLADE THICKNESS IN TWO SPECIES \\ OF LEUCAENA Benth. (LEGUMINOSAE) AND THEIR HYBRIDS
}

\section{NOTA}

\author{
Paulo Emílio Kaminski ${ }^{1}$ Maria Teresa Schifino -Wittmann²
}

RESUMO

A espessura da lâmina foliar foi analisada em duas espécies tetraplóides $(2 n=104)$ de Leucaena, L. leucocephala e L. diversifolia, e em híbridos entre ambas, num total de 25 plantas. Para cada planta, foram realizados cortes de 10 foliólulos. Os híbridos apresentaram valores médios para espessura da lâmina foliar intermediários $(116,3-148,3 \mu \mathrm{m})$ entre os das

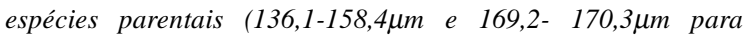
L.leucocephala e L.diversifolia, respectivamente) e uma menor variabilidade individual.

Palavras-chave : Leucaena, híbridos, espessura da lâmina foliar.

\section{SUMMARY}

The foliar blade thickness was analyzed in two tetraploid $(2 n=104)$ species of Leucaena, L. leuccocephala and L. diversifolia and in hybrids between both, in a total of 25 plants. For each plant, 10 leaflets were measured. The hybrids presented intermediate average values of foliar blade (116.3 $148.3 \mu \mathrm{m})$ in comparison with the parental species values (136.1$158.4 \mu \mathrm{m}$ and 169.2- $170.3 \mu \mathrm{m}$ for $\boldsymbol{L}$. leucocephala and $\boldsymbol{L}$. diversifolia, respectively) and a smaller invidual variation.

Key words : Leucaena, hybrids, foliar blade thickness

O gênero Leucaena, nativo da América Central e México, compreende árvores e arbustos multipropósito, algumas das quais com grande importância econômica especialmente no cinturão tropical do mundo (HUGHES, 1993; 1998). A utilização de algumas destas espécies, principalmente $\boldsymbol{L}$. leucocephala e L. diversifolia, como forrageiras em regiões de clima temperado e subtropical, como o Rio Grande do Sul, é extremamente promissora, desde que sejam selecionados materiais tolerantes ao frio e a solos ácidos. Neste sentido, um amplo projeto está sendo desenvolvido no Departamento de Plantas Forrageiras e Agrometeorologia da Faculdade de Agronomia da UFRGS, visando a selecionar germoplasma adaptado às condições do Estado. As abordagens do projeto são múltiplas, como caracterização e avaliação morfológica e de tolerância ao frio, análise citogenética, padrões de isoenzimas e produção estacional de forragem (FREITAS $\boldsymbol{e t}$ al., 1991 a e b; 1995; SCHIFINO-WITTMANN et al., 1996; SIMIONI, 1996)

Nesta nota, são apresentados os resultados da análise da variabilidade da espessura da lâmina foliar em indivíduos de L. leucocephala $(2 \mathrm{n}=104)$, L. diversifolia $(2 \mathrm{n}=104)$ e híbridos entre ambas as espécies, tendo por base uma amostra de 25 indiví-

\footnotetext{
${ }^{1}$ Biólogo, Mestre em Zootecnia, Universidade Federal do Rio Grande do Sul (UFRGS).

2 Biólogo, Professor Adjunto, Dr., Departamento de Plantas Forrageiras e Agrometeorologia, Faculdade de Agronomia, UFRGS, Caixa Postal 776, 91501-970, Porto Alegre, RS. Autor para correspondência. E-mail: $\underline{\text { mtschif@ vortex.ufrgs.br }}$ Recebido para publicação em 12.12.97. Aprovado em 09.12.98.
} 
duos de Leucaena, sendo 5 de L. leucocephala, 5 de L. diversifolia e 15 híbridos entre estas duas espécies. De cada indivíduo foram analisados 28 foliólulos e calculados a média e o desvio padrão. O n amostral foi obtido através do cálculo da média e desvio padrão, primeiramente entre dois foliólulos, e, posteriormente, foram acrescentados mais dois, novamente calculados a média e desvio padrão e, assim, sucessivamente até que o desvio padrão não mais se alterasse. Para a análise, os foliólulos, do quinto e sexto pares de cada ramo, foram cortados longitudinalmente com lâmina do tipo gilette, utilizando-se dois pedaços de cenoura como meio de suporte para o tecido a ser cortado. Os cortes eram armazenados em placas de petri com água destilada e, posteriormente, montados em lâminas para análise em microscópio ótico em aumento 10x100, com auxílio de ocular graduada. Para cada planta, foram analisados 10 cortes e para cada corte foram medidos a espessura total da lâmina, do parênquima paliçádico, do parênquima lacunoso e da epiderme. Os cortes eram medidos um pouco abaixo ou acima do feixe central de vasos condutores, dependendo qual deles apresentava melhor nitidez

Os resultados encontrados demonstram que existe uma diferença entre as amplitudes de variação das médias entre as duas espécies analisadas (tabela 1). As amplitudes das médias da espessura lâmina foliar foram menores em L. leucocephala (136,1 $158,4 \mu \mathrm{m})$ do que em $L$. diversifolia (169,2 $170,3 \mu \mathrm{m})$. Porém, a variação individual era maior em $\boldsymbol{L}$. leucocephala $(100-194 \mu \mathrm{m})$ do que em $\boldsymbol{L}$.

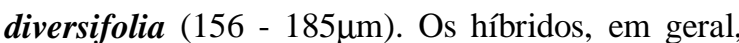
apresentavam médias intermediárias entre as duas

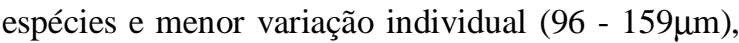
mas em alguns indivíduos as médias encontradas eram menores $(116,3 \mu \mathrm{m})$ do que as menores de $\boldsymbol{L}$. leucocephala $(136,1 \mu \mathrm{m})$. Houve sobreposição para a variação individual encontrada entre os três grupos: 100 a $194 \mu \mathrm{m}$ em L. leucocephala, 156 a $185 \mu \mathrm{m}$, em $\boldsymbol{L}$. diversifolia, e 94 , a $159 \mu \mathrm{m}$ para os híbridos. Para a variação média, foi encontrada sobreposição somente entre os grupos de $\boldsymbol{L}$.

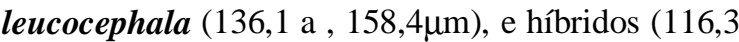
a $148,3 \mu \mathrm{m})$. Os resultados demonstram que há variação da espessura da lâmina foliar na população de Leucaena analisada. Como todos os indivíduos analisados eram tetraplóides, as diferenças não são explicáveis por níveis de ploidia diferentes.
Tabela 1 - Espessura da lâmina foliar em Leucaena leucocephala, L.diversifolia e híbridos (valores em $\mu \mathrm{m}$ )

\begin{tabular}{|c|c|c|c|}
\hline Espécie & $\begin{array}{c}\text { Número do } \\
\text { indivíduo }\end{array}$ & $\begin{array}{l}\text { Variação } \\
\text { Individual }\end{array}$ & $\begin{array}{c}\text { Variação } \\
\text { média }\end{array}$ \\
\hline \multirow[t]{5}{*}{ L.leucocephala } & 01 & $128-153$ & 139.6 \\
\hline & 02 & $100-152$ & 136.1 \\
\hline & 03 & $131-194$ & 158.4 \\
\hline & 04 & $133-161$ & 150.1 \\
\hline & 05 & $123-154$ & 137.9 \\
\hline \multirow[t]{5}{*}{ L.diversifolia } & 01 & $164-177$ & 169.8 \\
\hline & 02 & $161-179$ & 169.0 \\
\hline & 03 & $162-174$ & 169.2 \\
\hline & 04 & $158-181$ & 170.3 \\
\hline & 05 & $156-185$ & 170.0 \\
\hline \multirow[t]{15}{*}{ Híbridos } & 01 & $136-159$ & 147.9 \\
\hline & 02 & $137-151$ & 144.4 \\
\hline & 03 & $128-154$ & 144.1 \\
\hline & 04 & $136-156$ & 148.3 \\
\hline & 05 & $124-149$ & 140.7 \\
\hline & 06 & $131-156$ & 144.5 \\
\hline & 07 & $137-150$ & 145.7 \\
\hline & 08 & $133-147$ & 141.9 \\
\hline & 09 & $136-150$ & 143.0 \\
\hline & 10 & $122-146$ & 133.3 \\
\hline & 11 & $130-152$ & 141.3 \\
\hline & 12 & $133-155$ & 145.9 \\
\hline & 13 & $124-152$ & 138.8 \\
\hline & 14 & 096-133 & 116.3 \\
\hline & 15 & $103-136$ & 118.6 \\
\hline
\end{tabular}

\section{REFERÊNCIAS BIBLIOGRÁFICAS}

FREITAS, L.H.C. de, PAIM, N.R., SCHIFINO-WITTMANN, M.T. Morphological characterization and cytogenetical analysis of Leucaena leucocephala X tetraploid L. diversifolia. Leucaena Reserach Reports,Taiwan, v. 12, n. 11 , p. 16-18, nov. 1991(a).

FREITAS, L.H.C. de, SCHIFINO-WITTMANN, M.T., PAIM, N.R. Floral characteristics, chromosome number and meiotic behavior of hybrids between Leucaena leucocephala $(2 \mathrm{n}=104)$ and tetraploid $\boldsymbol{L}$. diversifolia $(2 \mathrm{n}=104)$ (Leguminosae). Revista Brasileira de Genética, Ribeirão Preto, v. 14, n. 3, p. 781-789, março, 1991(b).

FREITAS, L.H.C. de, PAIM, N.R., SCHIFINO-WITTMANN, M.T. Caracterização morfológica de híbridos de Leucaena leucocephala e Leucaena diversifolia. Revista Pesquisa 
Agropecuária Brasileira, Brasília, v. 30, n. 1, p. 61-68, jan. 1995.

HUGHES, C.E. Leucaena genetic resources: the OFI Leucaena seed collections and a synopsis of species characteristics. Oxford: Oxford Forestry Institute, 1993. 117 p.

HUGHES, C.E. Leucaena:a genetic resources handbook. Oxford: Oxford Forestry Institute, 1998. 280 p.

SCHIFINO-WITTMANN, FREITAS, L.H.C., SIMIONI, C. Isoenzymatic characterization of hybrids between Leucaena leucocephala and Leucaena diversifolia ssp. diversifolia grown in Rio Grande do Sul (Southern Brazil). Revista Brasileira de Genética, Ribeirão Preto, v. 19, n. 3, p. 475-478, junho 1996.

SIMIONI, C. Avaliação de tolerância ao frio e caracterização de híbridos entre Leucaena leucocephala e L. diversifolia ssp diversifolia. Porto Alegre, 1996. 108 p. Dissertação (Mestrado em Agronomia) - Curso de Pós-graduação em Zootecnia, Universidade Federal do Rio Grande do Sul, 1996.

Ciência Rural, v. 29, n. 4, 1999. 